\title{
Optimization of the production of biodiesel assisted by ultrasonic and microwave
}

\author{
Ming-Chien Hsiao" ${ }^{1}$, Chin-Chiuan Lin ${ }^{2, *}$ \\ ${ }^{1}$ Department of Environment Engineering, Kun Shan University, Taiwan, R.O.C \\ ${ }^{2}$ Department of Business Administration, Kun Shan University, Taiwan, R.O.C
}

\section{Email address:}

johnson@mail.ksu.edu.tw (Chin-Chiuan Lin), cclin@mail.ksu.edu.tw (Chin-Chiuan Lin)

\section{To cite this article:}

Ming-Chien Hsiao, Chin-Chiuan Lin. Optimization of the Production of Biodiesel Assisted by Ultrasonic and Microwave, International Journal of Energy and Power Engineering. Vol. 2, No. 2, 2013, pp. 54-59. doi: 10.11648/j.ijepe.20130202.14

\begin{abstract}
The transesterification reaction of present study was assisted by ultrasonic mixing and microwave irradiation. Central composite design (CCD) was employed to optimize the transesterification reaction conditions of biodiesel production from soybean oil. A mathematical model predicted that the theoretical conversion rate of soybean oil of $102.19 \%$ could be obtained under the following reaction conditions: amount of catalyst used, $1.4 \mathrm{wt} \%$; reaction temperature, $57.89^{\circ} \mathrm{C}$; and methanol/oil molar ratio, 8.11:1, assisted by 1-min ultrasonic mixing and 2-min microwave irradiation. This result indicated that there are a wide range of reaction conditions to obtain conversion rate approach $100 \%$ assisted by ultrasonic mixing and microwave irradiation. Combined the ultrasonic mixing and microwave irradiation are very efficiency process to produce biodiesel. The total reaction time was shorter than those obtained in previous studies.
\end{abstract}

Keywords: Catalyst, Reaction Temperature, Methanol/Oil Molar Ratio, Ultrasonic, Microwave, Soybean Oil

\section{Introduction}

Biodiesel shows numerous advantages including decreased greenhouse gas emissions, lower sulfide emission, being biodegradable and non-toxic fuel, and possible production from agricultural surplus that can also help improve rural economies [1,2]. Soybean oil is one of the most important vegetable oils for producing biodiesel due to its high oil content (around 20\%). Considerable studies have been done on soybean oil for biodiesel (fatty acid melkyl esters, FAMEs) production.

The transesterification of soybean oil into biodiesel using the traditional mechanical stirring process under a high temperature (above $70^{\circ} \mathrm{C}$ ) and an alcohol-to-oil ratio of $6: 1$ has been previously reported by Diasakou et al. [3] and Noureddini et al. [4]. Today, ultrasonic mixing [5-7] and microwave irradiation [8-10] are widely used in assisting transesterification of vegetable oils to produce biodiesel. Previous studies indicated that ultrasonic mixing is efficient, time-saving and economically functional, offering a lot of advantages over the classical procedure [6,7]. Meanwhile, microwave irradiation has been an alternative heating system for transesterification over the past few years [8]. Microwave irradiation has achieved a drastic reduction in the quantity of by-products and a shorter separation time
[9]. In addition, it also provides a comfortable, safe and clean way of carrying out chemical reactions [10].

Most previous researches focused on transesterification assisted only by either ultrasonic mixing or microwave irradiation, studies on transesterification assisted by both ultrasonic mixing and closed microwave irradiation are relatively scarce.

According to previous studies, the amount of catalyst, reaction temperature, and methanol/oil molar ratio were significant factors affecting conversion rate of vegetable oil $[11,12]$. Therefore, hypothetically, they might be significant factors influencing conversion rate of soybean oil, and their optimal reaction levels should be approximately as follows: amount of catalyst used, $1.0 \mathrm{wt} \%$; reaction temperature, $60^{\circ} \mathrm{C}$; and methanol/oil molar ratio, 6:1 [13].

The objectives of the present study were to evaluate the influence of amount of catalyst used, reaction temperature, and methanol/oil molar ratio on soybean oil conversion into FAMEs, and to investigate their optimal reaction level under the assistance of ultrasonic mixing and microwave irradiation.

\section{Methods}

\subsection{Materials}


The present study used refined commercial soybean oil whose water and free fatty acid (FFA) contents were about $0.11 \%$ and $0.03 \%$, respectively. The alcohol and catalyst used in the present study were methanol and $\mathrm{NaOH}$, respectively. Methanol $99.8 \%$ and $\mathrm{NaOH} 98 \%$ were purchased from Shimakyu Co. Ltd. (Japan) and Mallinckrodt Co. Ltd. (USA), respectively. Methyl laurate and acetate were purchased from Fluka Co. Ltd. (USA).

\subsection{Equipment}

Ultrasonic equipment used in the experimental had a working frequency of $20 \mathrm{kHz}$ (Mixsonix Sonicator 3000) and an output power of $600 \mathrm{~W}$.

The experiment was also conducted using a commercial type microwave apparatus (Milestone ETHOS 900). Figure 1 shows the setup of microwave irradiation assisted transesterification. The machine comprised a continuous microwave power delivery system, with a working frequency of $2.45 \mathrm{GHz}$, power output from 0 to $900 \mathrm{~W}$, and the temperature and time setting.

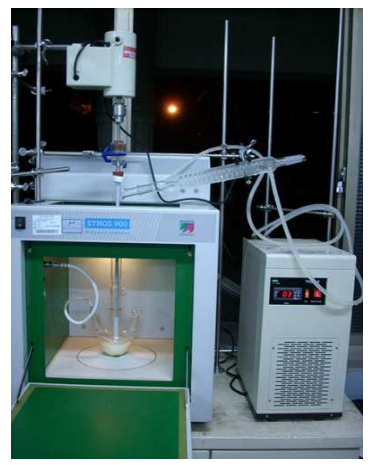

Figure 1. Setup of microwave irradiation assisted transesterification.

\subsection{Transesterification Process}

Triglycerides were converted into esters with methanol using alkali catalyst. The transesterification involves two discrete steps.

In the first step, reaction of reagents was assisted by ultrasonic mixing. The temperature during ultrasonic mixing was about $30-32^{\circ} \mathrm{C}$ (just about $2^{\circ} \mathrm{C}$ higher than room temperature after ultrasonic mixing). The spherical glass reactors had volumes of $250 \mathrm{ml}$ that was modified by the introduction of a water condenser for conducting atmospheric pressure experiments.

In the second step, reaction of reagents was assisted by microwave irradiation. The reaction temperature was set in this step.

After the reaction, the mixture was allowed to settle for half an hour and the FAME (or methanol-water) fraction that separated at the top was removed in a separating funnel.

\subsection{Analytical Methods}

The acid value ( $\mathrm{AV}, \mathrm{KOH} \mathrm{mg} / \mathrm{g}$ ) and saponification value ( $\mathrm{SV}, \mathrm{KOH} \mathrm{mg} / \mathrm{g}$ ) were determined by a standard titrimetry method (AOCS: American Oil Chemists' Society). The molecular weight (MW) of soybean oil was calculated from its saponification value (SV) and acid value (AV). The experimental results of $\mathrm{AV}, \mathrm{SV}$ and MW were $0.06,194.98$ and 863.31 , respectively.

After reaction, the top layer contained mainly FAMEs. Having settled for half an hour, the FAMEs were removed in a separating funnel, washed at least three times with acetate $30 \%$ and deionized water, and finally dried in an oven at $105 \pm 3{ }^{\circ} \mathrm{C}$.

The samples were analyzed with a Perkin Elmer GC Clarus 600 equipped with a capillary column (SPBTM-WAX, $30 \mathrm{~m} \times 0.75 \mathrm{~m} \times 1.0 \mu \mathrm{m})$ and a flame ionization detector (FID).

Methyl laurate was added as an internal standard into the crude biodiesel and the sample was injected under the following conditions: carrier gas, nitrogen; injector temperature, $280^{\circ} \mathrm{C}$; split ratio, 1:20; and temperature of detector, $300^{\circ} \mathrm{C}$. Oven temperature started at $210^{\circ} \mathrm{C}$ for $2 \mathrm{~min}$, increased to $240^{\circ} \mathrm{C}$ at a rate of $4^{\circ} \mathrm{C} / \mathrm{min}$ and held for $8 \mathrm{~min}$.

The conversion rate of crude biodiesel was calculated according to the area of FAME as expressed in the following equation [13]:

$$
\begin{gathered}
\text { Conversion rate }(\%)= \\
\left(\frac{\text { area of } F A M E \div \text { area of reference } \times \text { weight of reference }}{\text { weight of crude biodiesel }}\right) \times 100
\end{gathered}
$$

\subsection{Ultrasonic Mixing and Microwave Irradiation Process}

The effects of ultrasonic mixing and microwave irradiation on the conversion rate of soybean oil are shown in Table 1. The constant reaction conditions were amount of catalyst, $1.0 \mathrm{wt} \%$; reaction temperature, $60^{\circ} \mathrm{C}$; and methanol/oil molar ratio, 6:1. All designed experiment combinations were conducted in triplicate. Table 1 shows that the mean conversion rates achieved at 1 and $2 \mathrm{~min}$ by microwave irradiation alone without ultrasonic mixing (ultrasonic mixing 0 min) were only 11.24 and $13.31 \%$, respectively. When ultrasonic mixing of $0.5 \mathrm{~min}$ was added to the procedure, the conversion rates at 1- and 2-min microwave irradiation rose to 50.03 and $57.13 \%$, respectively. Further, the conversion rates were significantly high with ultrasonic mixing for 1 min followed by microwave irradiation for $1(91.64 \%)$ and 2 $\min (97.63 \%)$, because ultrasonic mixing causes cavitation of bubbles near the phase boundary between the soybean oil and methanol [5]. In short, if the soybean oil and methanol were not mixed thoroughly, the conversion rate would be very low. On the contrary, if the soybean oil and methanol were mixed thoroughly after ultrasonic mixing for $1 \mathrm{~min}$, the conversion rate achieved by 2-min closed microwave irradiation would become very high $(97.63 \%)$. According to the standard of EN-14214 (In order for biodiesel to be used as a motor fuel or blended with petroleum diesel, it must comply with standard specifications), the conversion rate of methyl esters should be $96.4 \%$. Hence, the most time-saving process should be 1-min ultrasonic mixing, followed by 2-min microwave irradiation. 
Table 1. Conversion rates of soybean oil under assistance of ultrasonic mixing and microwave irradiation.

\begin{tabular}{lll}
\hline $\begin{array}{l}\text { Ultrasonic mixing time } \\
\text { (min) }\end{array}$ & $\begin{array}{l}\text { microwave irradiation time } \\
(\mathbf{m i n})\end{array}$ & $\begin{array}{l}\text { Conversion rate } \\
(\mathbf{\%})\end{array}$ \\
\hline 0.0 & 1.0 & 11.24 \\
& 2.0 & 13.31 \\
0.5 & 1.0 & 50.03 \\
& 2.0 & 57.13 \\
1.0 & 1.0 & 91.64 \\
& 2.0 & 97.63 \\
\hline
\end{tabular}

After reaction, the top layer contained mainly FAMEs. Having settled for half an hour, the FAMEs were removed in a separating funnel, washed at least three times with acetate $30 \%$ and deionized water, and finally dried in an oven at $105 \pm 3^{\circ} \mathrm{C}$.

\subsection{Experimental Design}

Central composite design (CCD) was used in the production of FAMEs, which is generally the best design for response surface optimization [14].

A five-level-three-factor design was selected. The three variables selected were reaction temperature, amount of catalyst, and methanol/oil molar ratio, and their respective levels were as follows: reaction temperature $\left(x_{1}\right.$, 51.59-68. $\left.41^{\circ} \mathrm{C}\right)$, amount of catalyst $\left(x_{2}, 0.16-1.84 \mathrm{wt} \%\right)$, and methanol/oil molar ratio $\left(x_{3}, 0.95: 1-11.05: 1\right)$. Table 2 shows the coded and uncoded levels of independent variables.

Table 2. Coded and uncoded levels of independent variables for CCD.

\begin{tabular}{|c|c|c|c|c|c|}
\hline \multirow{2}{*}{$\begin{array}{l}\text { Independent varia- } x_{i} \\
\text { ble }\end{array}$} & \multicolumn{5}{|c|}{ Codes factor levels } \\
\hline & $-\alpha$ & -1 & $\mathbf{0}$ & +1 & $+\alpha$ \\
\hline $\begin{array}{ll}\text { Reaction } & \text { tempera- } \\
\text { ture } & \end{array}$ & 51.59 & 55 & 60 & 65 & 68.41 \\
\hline Catalyst amount & 0.16 & 0.5 & 1.0 & 1.5 & 1.84 \\
\hline $\begin{array}{l}\text { Methanol/oil molar } \\
\text { ratio }\end{array}$ & 0.95 & 3 & 6 & 9 & 11.05 \\
\hline
\end{tabular}

Each response of the reaction was employed to develop a mathematical model that correlates the yield of soybean oil FAMEs with the transesterification process variables studied through first-order, second-order, and interaction terms, according to the following second-order equation [14].

$$
\beta_{0}+\sum_{i=1}^{3} \beta_{i} x_{i}+\sum_{i=1}^{3} \beta_{i i} x_{i}{ }^{2}+\sum_{i=1}^{2} \sum_{j=i+1}^{3} \beta_{i j} x_{i} x_{j}^{\mu}+
$$

where $\mu$ is the predicted response, conversion rate ( $\%)$. The coefficient $\beta_{0}$ is the free or offset term called intercept. Coefficients $\beta_{i}, \beta_{i i}$, and $\beta_{j i}$ signify respectively first-order, quadratic-order, and first-order interaction effects for the variables. The term $r_{i}$ allows for uncertainties or discrepancies between predicted value and measured value of re- sponse, and stands for the residual [15]. $x_{1}, x_{2}$, and $x_{3}$ are the coded values of independent factors, reaction temperature, amount of catalyst, and methanol/oil molar ratio, respectively.

\section{Results and Discussion}

All 20 of the designed experiment combinations were conducted, which included eight factorial points, six axial points, and six central points to provide information regarding the interior of the experiment region, allowing for the evaluation of the curvature $[14,15]$. All experiment combinations were also carried out in triplicate. The experimental results are summarized in Appendix Table 1. The mean conversion rate ranged from $15.32 \%$ to $97.78 \%$, depending on the experimental conditions.

Appendix Table 1. Central composite second-order design, experimental

\begin{tabular}{|c|c|c|c|c|c|}
\hline $\begin{array}{l}\text { St } \\
\text { d }\end{array}$ & $\begin{array}{l}\mathbf{R u} \\
\mathbf{n}\end{array}$ & $\begin{array}{l}\text { Reaction } \\
\text { temperature }\end{array}$ & $\begin{array}{l}\text { Catalyst } \\
\text { amount }\end{array}$ & $\begin{array}{l}\text { Methanol/oil } \\
\text { molar ratio }\end{array}$ & $\begin{array}{l}\text { Conversion } \\
\text { rate }(\%)\end{array}$ \\
\hline 1 & 17 & $-1(55)$ & $-1(0.5)$ & $-1(3: 1)$ & 44.95 \\
\hline 2 & 8 & $+1(65)$ & $-1(0.5)$ & $-1(3: 1)$ & 49.83 \\
\hline 3 & 12 & $-1(55)$ & $-1(0.5)$ & $+1(9: 1)$ & 49.95 \\
\hline 4 & 9 & $+1(65)$ & $-1(0.5)$ & $+1(9: 1)$ & 45.85 \\
\hline 5 & 16 & $-1(55)$ & $+1(1.5)$ & $-1(3: 1)$ & 37.89 \\
\hline 6 & 13 & $+1(65)$ & $+1(1.5)$ & $-1(3: 1)$ & 32.68 \\
\hline 7 & 7 & $-1(55)$ & $+1(1.5)$ & $+1(9: 1)$ & 81.84 \\
\hline 8 & 1 & $+1(65)$ & $+1(1.5)$ & $+1(9: 1)$ & 83.59 \\
\hline 9 & 11 & $-\alpha(51.59)$ & $0(1)$ & $0(6: 1)$ & 75.84 \\
\hline 10 & 15 & $+\alpha(68.41)$ & $0(1)$ & $0(6: 1)$ & 75.23 \\
\hline 11 & 3 & $0(60)$ & $0(1)$ & $-\alpha(0.95)$ & 25.64 \\
\hline 12 & 6 & $0(60)$ & $0(1)$ & $+\alpha(11.05)$ & 78.01 \\
\hline 13 & 2 & $0(60)$ & $-\alpha(0.16)$ & $0(6: 1)$ & 15.32 \\
\hline 14 & 4 & $0(60)$ & $+\alpha(1.84)$ & $0(6: 1)$ & 79.72 \\
\hline 15 & 5 & $0(60)$ & $0(1)$ & $0(6: 1)$ & 97.59 \\
\hline 16 & 10 & $0(60)$ & $0(1)$ & $0(6: 1)$ & 97.68 \\
\hline 17 & 18 & $0(60)$ & $0(1)$ & $0(6: 1)$ & 97.68 \\
\hline 18 & 14 & $0(60)$ & $0(1)$ & $0(6: 1)$ & 97.35 \\
\hline 19 & 20 & $0(60)$ & $0(1)$ & $0(6: 1)$ & 97.68 \\
\hline 20 & 19 & $0(60)$ & $0(1)$ & $0(6: 1)$ & 97.78 \\
\hline
\end{tabular}
and predicted data for five-level-three-factor response surface analysis.

\subsection{Effects of Variables in Transesterification Process}

As seen in Appendix Table 2, among the three individual variables studied, methanol/oil molar ratio $\left(x_{3}\right)$ has the largest effect on the FAME conversion rate of soybean oil (due to the highest $F$-value) followed by amount of catalyst $\left(x_{2}\right)$, and reaction temperature $\left(x_{1}\right)$.

Figure 2 to 4 show the isoresponse surface plots for the 
transesterification conditions of FAME production. Further, Figure 2 and 4 shows the maximum predicted theoretical conversion rate was greater than $100 \%$. This result indi- cated that there are a wide range of reaction conditions to obtain conversion rate $100 \%$.

Appendix Table 2. ANOVA for response surface quadratic model on conversion rate of the independent factors.

\begin{tabular}{|c|c|c|c|c|c|}
\hline Source & Sum of squares & Degrees of freedom & Mean square & F-value & Prob $>$ F \\
\hline Model & 13930.22 & 9 & 1547.80 & 24.02 & $<0.0001$ \\
\hline$x_{2}$, Catalyst amount & 1844.82 & 1 & 1844.82 & 28.63 & $<0.0001$ \\
\hline$x_{3}$, methanol/oil molar ratio & 2614.38 & 1 & 2614.38 & 40.57 & 0.0001 \\
\hline$x_{1} \times x_{2}$ & 0.072 & 1 & 0.072 & 0.001 & 0.9740 \\
\hline$x_{1} \times x_{3}$ & 1.11 & 1 & 1.11 & 0.017 & 0.8982 \\
\hline$x_{2} \times x_{3}$ & 1221.17 & 1 & 1221.17 & 18.95 & 0.0014 \\
\hline$x_{1}^{2}$ & 961.32 & 1 & 961.32 & 14.92 & 0.0031 \\
\hline$x_{2}^{2}$ & 4706.77 & 1 & 47.6077 & 73.04 & $<0.0001$ \\
\hline$x_{3}^{2}$ & 3947.35 & 1 & 3947.35 & 61.25 & $<0.0001$ \\
\hline Residual error & 644.44 & 10 & 64.44 & & \\
\hline Lack-of-fit & 643.60 & 5 & 128.72 & 769.31 & $<0.0001$ \\
\hline Pure Error & 0.84 & 5 & 0.017 & & \\
\hline Cor Total & 14574.66 & 19 & & & \\
\hline
\end{tabular}

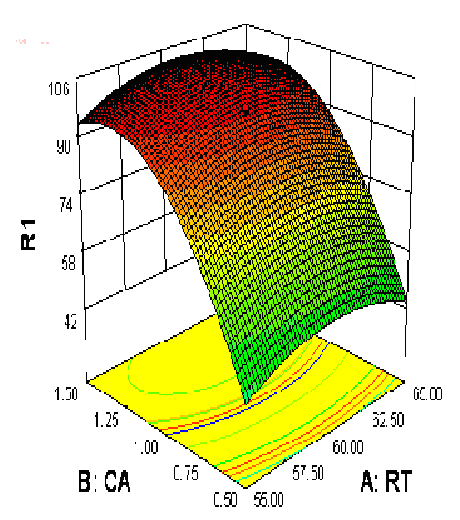

Figure 2. Interaction effect between reaction temperature (RT) and amount of catalyst (CA) on biodiesel conversion rate.

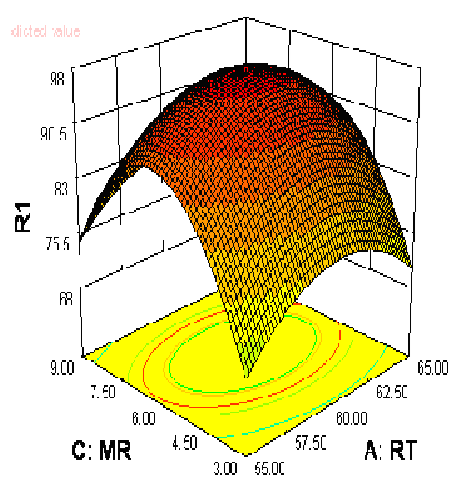

Figure 3. Interaction effect between reaction temperature $(R T)$ and methanol/oil molar ratio (MR) on biodiesel conversion rate.

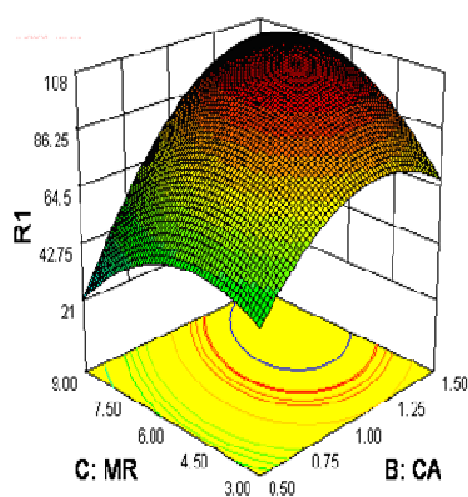

Figure 4. Interaction effect between amount of catalyst (CA) and methanol/oil molar ratio (MR) on biodiesel conversion rate.

The maximum predicted theoretical conversion rate of $102.19 \%$ was obtained at reaction temperature of $57.89^{\circ} \mathrm{C}$, amount of catalyst of $1.4 \mathrm{wt} \%$, and methanol/oil molar ratio of 8.11:1. The methanol/oil molar ratio was greater than previous studies [11-13]. This result can also explain why the maximum predicted theoretical conversion rate greater than previous studies [16].

\subsection{Development of Regression Model Equation}

The analysis of variance (ANOVA) results for the response surface quadratic model are shown in Appendix Table 2.

The model F-value of 24.02 implies that the model is significant. The p-value is less than 0.0001 , i.e., there is less than $0.01 \%$ chance that this error is caused by noise, im- 
plying a very high significance of the regression model [14]. The goodness-of-fit of the model is verified by the determination coefficient $\left(\mathrm{R}^{2}\right)$. In this case, the value of $\mathrm{R}^{2}(=$ 0.95 ) indicated that the model does not explain only $5 \%$ of the total variation. The value of adjusted determination coefficient (Adj $\mathrm{R}^{2}=0.91$ ) is also high for obtaining a high significance of the model [15].

The results were evaluated via multiple regression analysis and tested for significance. Finally, the best-fitting model was determined via regression, which showed that two linear coefficients $\left(\mathrm{x}_{2}, \mathrm{x}_{3}\right)$, one cross-product coefficients $\left(\mathrm{x}_{2} \mathrm{x}_{3}\right)$, and three quadratic coefficients $\left(\mathrm{x}_{1}{ }^{2}, \mathrm{x}_{2}{ }^{2}, \mathrm{x}_{3}{ }^{2)}\right.$ were significant.

The application of RSM [16] yields the following regression equation, which is an empirical relationship between the conversion rate of biodiesel and the test variables in the uncoded unit:

$$
\begin{array}{r}
\mu= \\
-1219.70+39.22 \chi_{1}+118.40 \chi_{2}+ \\
18.44 \chi_{3}-0.33 \chi_{1}{ }^{2}-72.29 \chi_{2}{ }^{2}-1.84 \chi_{3}{ }^{2}+8.24 \chi_{2} \chi_{3}
\end{array}
$$

The predicted optimum levels of reaction temperature, amount of catalyst, and methanol/oil molar ratio of the transesterification reaction were obtained by applying the regression analysis to equation (3). The positive sign in front of the terms indicates synergistic effect, while the negative sign indicates antagonistic effect. Equation (3) shows that the FAME conversion rate of soybean oil has linear, quadratic and interaction effects on the three variables studied.

Figure 5 shows that there are a wide range of reaction conditions to obtain conversion rate approach $100 \%$ assisted by 1-min ultrasonic mixing and 2-min microwave irradiation.

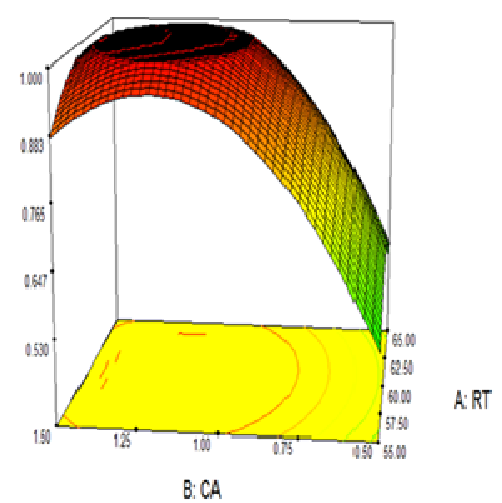

Figure 5. Isoresponse surface of predicted conversion rate.

\subsection{Validation Experiments}

The present study also performed validation experiments in triplicate to confirm the optimum reaction conditions: reaction temperature, $57.89^{\circ} \mathrm{C}$; amount of catalyst, $1.4 \mathrm{wt} \%$; and methanol/oil molar ratio, 8.11:1. Results showed that the mean conversion rate was $98.44 \%$, which was slightly lower than that predicted by the model. Nevertheless, the model was validated by conducting experiments at optimal design conditions.

\subsection{Total Reaction Time and Conversion Rate}

Previous studies have also employed RSM to evaluate the influence of alcohol-to-oil molar ratio and catalyst concentration on the yield of conversion of soybean oil into ethyl esters; comparatively, the conversion rate of the present study was slightly lower than that obtained by Santos et al. [5]. However, the total reaction time (3 $\mathrm{min}$ ) of the present study was shorter than that obtained by Santos et al. [5], which was $30 \mathrm{~min}$ when assisted by ultrasound mixing. Further, the conversion rate obtained in the present study was higher than that obtained by Rodrigues et al. [17] while the total reaction time of the present study was also shorter than that observed by Rodrigues et al. [17], which was also 30 min when assisted by ultrasound mixing.

\section{Conclusions}

In the present study, $\mathrm{CCD}$ was employed to optimize the transesterification condition of biodiesel from soybean oil assisted by ultrasonic mixing and microwave irradiation. A mathematical model predicted that the theoretical conversion rate of soybean oil of $102.19 \%$ can be obtained under the following optimum reaction conditions: reaction temperature of $57.89^{\circ} \mathrm{C}$, amount of catalyst of $1.4 \mathrm{wt} \%$, and methanol/oil molar ratio of $8.11: 1$, assisted by 1 -min ultrasonic mixing and 2-min microwave irradiation. Results indicated that there are a wide range of reaction conditions to obtain conversion rate approach $100 \%$ assisted by ultrasonic mixing and microwave irradiation. Combined the ultrasonic mixing and microwave irradiation are very efficiency process to produce biodiesel. The total reaction time was shorter than those obtained in previous studies. The quality of biodiesel produced from soybean oil under the optimum reaction conditions satisfied the relevant quality standards and the total reaction time was shorter than those obtained in previous studies.

\section{Acknowledgements}

This study was supported by a research grant from the National Science Council of the Republic of China, grant no. NSC 98-2622-E-168-018-CC3.

\section{References}

[1] G. Vicente, M. Martınez, and J. Aracil, "Integrated biodiesel production: A comparison of different homogeneous catalysts systems," Bioresource Technology, vol. 92, 2004, pp.297-305.

[2] D. E. Lopez, J. G. Goodwin, D. A. Bruce, and E. Lotero, "Transesterification of triacetin with methanol on solid acid and base catalysts," Applied Catalysis A: General, vol. 295, 2005, pp. 97-105. 
[3] M. Diasakou, A. Louloudi, and N. Papayannakos, "Kinetics of the non-cataytic transesterification of soybean oil," Fuel, vol. 77, 1998, pp. 1297-1302.

[4] H. Noureddini, V. Harkey, and V. Medikondure, "A continuous process for the conversion of vegetable oils into methyl esters of fatty acids," Journal of the American Oil Chemists Society, vol. 75, 2005, pp. 1775-1783.

[5] F. F. P. Santos, S. Rodrigues, and A. N. Fernandes, "Optimization of the production of biodiesel from soybean oil by ultrasound assisted methanolysis," Fuel Processing Technology, vol. 90, 2009, pp. 312-316.

[6] C. Stavarache, M. Vinatoru, R. Nishmura, and Y. Maeda, "Fatty acids methyl esters from vegetable oil by means of ultrasonic energy," Ultrasonics Sonochemistry, vol. 12, 2005, pp. 367-372.

[7] J. Ji, J. Wang, Y. Li, Y. Yu, and Z. Xu, "Preparation of biodiesel with the help of ultrasonic and hydrodynamic cavitation," Ultrasonics, vol. 44, 2006, pp. e411-e414.

[8] N. Azcan, and A. Danisman, "Microwave assisted transesterification of rapeseed oil," Fuel, vol. 87, 2008, pp.1781-1788.

[9] C. Koopmans, M. Iannelli, P. Kerep, M. Klink, S. Schmitz, S. Sinnwell, and H. Ritter, "Microwave-assisted polymer chemistry: Heck-reaction, transesterification, baeyer-villiger oxidation, oxazoline polymerization, acrylamides, and porous materials," Tetrahedron, vol. 62, 2006, pp. 4709-4714.
[10] J. Hernando, P. Leton, M. P. Matia, J. L. Novella, and J. Alvarez-Builla, "Biodiesel and fame synthesis assisted by microwaves: Homogeneous batch and flow process," Fuel, vol. 86, 2006, pp. 1641-1644.

[11] Y. C. Sharma, B. Singh, and J. Korstad, "Latest developments on application of heterogenous basic catalysts for an efficient and eco friendly synthesis of biodiesel: A review," Fuel, vol. 90, 2011, pp. 1309-1324.

[12] D. Y. C. Leung, X. Wu, and M. K. H. Leung, "A review on biodiesel production using catalyzed transesterification," Applied Energy, vol. 87, 2010, pp. 1083-1095.

[13] M.-C. Hsiao, C.-C. Lin, and Y.-H. Chang, "Microwave irradiation-assisted transesterification of soybean oil to biodiesel catalyzed by nanopowder calcium oxide," Fuel, vol. 90, 2011, pp. 1963-1967.

[14] G. E. P. Box, and N. R. Draper, Empirical model building and response surfaces, John Wiley \& Sons, New York, 1986.

[15] D. C. Montgomery, Design and analysis of experiments, 3rd ed., John Wiley \& Sons, New York, 1991.

[16] S. Rodrigues, L. C. A. Mazzone, F. F. P. Santos, M. G. A. Cruz, and F. A. N. Fernandes, "Optimization of the production of ethyl esters by ultrasound assisted reaction of soybean oil and ethanol," Brazilian Journal of Chemical Engineering, vol. 26, 2009, pp. 361-366.

[17] A. I. Khuri, and J. A. Cornell, Response surfaces, 2nd Edition, Dekker, New York, 1996. 